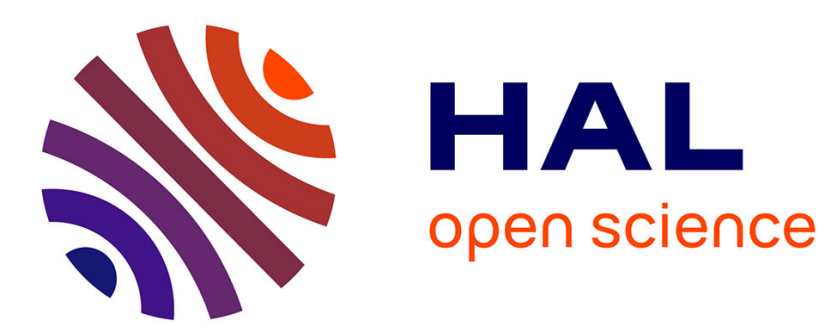

\title{
Multi-scale approach to cracking criteria for drying soils
}

Liang Bo Hu, Mohammad Monfared, Boleslaw Mielniczuk, Lyesse Laloui, Tomasz Hueckel, Moulay Saïd El Youssoufi

\section{To cite this version:}

Liang Bo Hu, Mohammad Monfared, Boleslaw Mielniczuk, Lyesse Laloui, Tomasz Hueckel, et al.. Multi-scale approach to cracking criteria for drying soils. Geo-Congress 2013, Mar 2013, San Diego, United States. pp.838-845, 10.1061/9780784412787.085 . hal-00835090

\section{HAL Id: hal-00835090 https://hal.science/hal-00835090}

Submitted on 18 Jun 2013

HAL is a multi-disciplinary open access archive for the deposit and dissemination of scientific research documents, whether they are published or not. The documents may come from teaching and research institutions in France or abroad, or from public or private research centers.
L'archive ouverte pluridisciplinaire HAL, est destinée au dépôt et à la diffusion de documents scientifiques de niveau recherche, publiés ou non, émanant des établissements d'enseignement et de recherche français ou étrangers, des laboratoires publics ou privés. 


\title{
Multi-scale approach to cracking criteria for drying silty soils
}

\author{
Liang Bo Hu${ }^{1}$, Mohammad Monfared ${ }^{2}$, Boleslaw Mielniczuk ${ }^{4}$, Lyesse Laloui ${ }^{2}$, \\ Tomasz Hueckel ${ }^{3}$, M. ASCE, Moulay Said El Youssoufi ${ }^{4}$ \\ ${ }^{1}$ University of Toledo, Toledo, OH 43606, USA, liangbo.hu@utoledo.edu \\ ${ }^{2}$ EPFL, Lausanne, CH-1015, Station 18, Switzerland; mohammad.monfared@epfl.ch; lyesse.laloui@epfl.ch \\ 3 Duke University, Durham, NC 27708, USA, hueckel@ duke.edu \\ ${ }^{4}$ Université Montpellier 2, 34095 Montpellier Cedex 5, France, boleslaw.mielniczuk@ univ-montp2.fr; $\underline{\text { Moulay-Said.El- }}$ \\ Youssoufi@univ-montp2.fr
}

\begin{abstract}
Cracking is a most unwanted development in soil structures undergoing periodic drying. Desiccation cracking arises in the apparent absence of external forces. Hence, either an internal, self equilibrated stress pattern resulting from kinematic incompatibilities, or stress resulting from reaction forces at the constraints should be contemplated to arrive at cracking criteria. Three circumstances are considered for drying cracks: drying shrinkage, kinematic constraints impeding the shrinkage inducing reaction forces, and consequent tensile effective stress reaching tensile strength. An earlier tubular micro-scale model of porous drying medium is considered with constrained at restrictive inter - pore solid contacts. At the mesoscale tubular drying pores are considered in the vicinity of an imperfection, inducing a stress concentration near its tip, in the presence of significant pore suction. This approach allows one to use the effective stress analysis, which otherwise, away from the stress concentration usually yields compressive effective stress and hence a physically incompatible criterion. Recent experimental results from an idealized configuration of a cluster of grains provide geometrical data suggesting that an imperfection as a result of air entry deep into the granular medium penetrates over 8 internal radii of the typical pore.
\end{abstract}

\section{INTRODUCTION}

Cracking is a highly undesirable occurrence in soil structures subject to drying. Desiccation cracking similarly to thermal cracking arises in the apparent absence of external forces. Therefore, either an internal, self-equilibrated stress pattern due to kinematic incompatibilities, or stress resulting from reaction forces at the constraints should be contemplated to arrive at a cracking criterion. The following conditions need to be considered for drying cracks: drying shrinkage, kinematic constraints impeding the shrinkage which induce 
reaction forces, and consequent tensile effective stress reaching tensile strength. An earlier tubular microscale model of porous drying medium can be considered in several configurations: unconstrained, completely constrained at the contact with the material matrix, and constrained at restrictive inter - pore solid contacts. The latter one is addressed in this paper. At the meso-scale tubular drying pores are considered in the vicinity of an imperfection, inducing a stress concentration near its tip, in the presence of significant pore suction. This approach allows one to use the effective stress analysis, which otherwise, away from the stress concentration usually yields compressive effective stress and hence a physically incompatible criterion. Experimental results from an idealized configuration of a cluster of grains provide geometrical data suggesting that an imperfection as a result of air entry deep into the granular medium penetrates over 8 internal radii of the typical pore.

\section{EXPERIMENTAL BACKROUND}

Recent microscale experiments with drying clusters of smooth glass grains (Mielniczuk et al., 2013) reveal that a non-symmetric mode of the unstable evolution of the water body leads at some point constitutes a most common air entry mechanism into the body of the saturated soil. While the circumstances of the event are not yet fully understood, it clearly is an unstable and localized displacement of the gas/liquid interface occurring at an order of magnitude faster rate than the preceding process manifested among others by a slow evolution of the liquid/gas interface, and involving dynamic effect from the released energy. Fig. 1 shows three of a series of images of a 2D saturated grain cluster (Mielniczuk et al., 2013) shot every 90 seconds during natural isothermal drying (using Canon EOS 500D). Grains were $3.5 \mathrm{~mm}$ in diameter ultrafine glass spheres, while the liquid is ultra-pure deionized water. As indicated, it took 10 minutes from the initial configuration to reach the intermediate one, and less than 1 additional minute to develop the final configuration with the gas penetration. The depth of the penetration of the gas finger is about 4 times the average size (diameter) of the pore.

The images posterior to the air incursion show a wave of motion of the mass of water lasting over several seconds displaced from the space occupied now by the gas finger.

It is to be noted that a similar scenario of the air entry has been observed in grain systems of 3, 4, 5, and 6 spheres. It needs also to be mentioned that the presented non-symmetric incursion of air is not the only scenario of air entry. An alternative entry mode takes place at a center point between the spheres and consists of a multi- step process: starting with a convergence of two gas/liquid interfaces situated opposite one another (at the front and back of the picture), followed by formation of a suspended thin film of liquid, followed by a further thinning of the film, leading to coalescence of the two surfaces, producing a short cylindrical or spherical gas nucleus, which subsequently propagates symmetrically with a circular projection until its boundary reaches the sold walls of the sphere. This is shown in Fig. 2 for a six-sphere cluster. Notably, the non-symmetrical scenario occurs for systems with higher separations between grains, while for lower separations a symmetrical scenario takes place. The former one takes place at a location of a negative Gaussian curvature of the meniscus, while the latter is associated with a positive Gaussian curvature (Mielniczuk et al., 2013). 


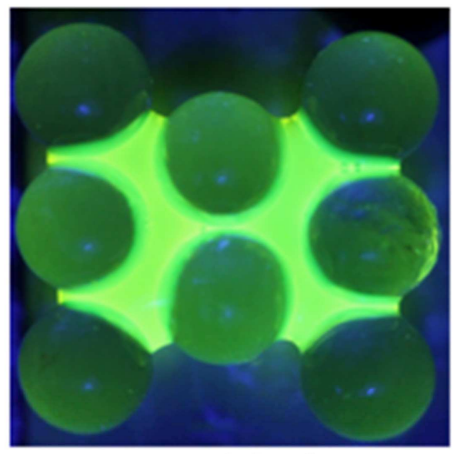

$0.0 \mathrm{~min}$

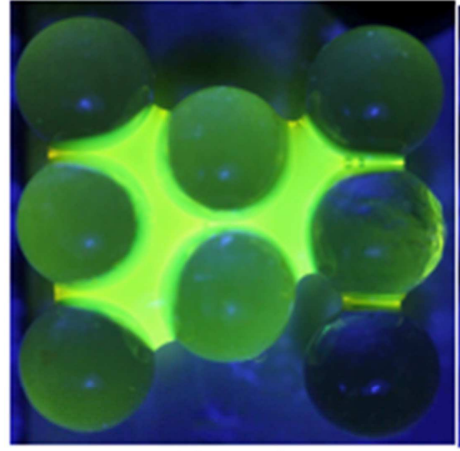

$10.0 \mathrm{~min}$

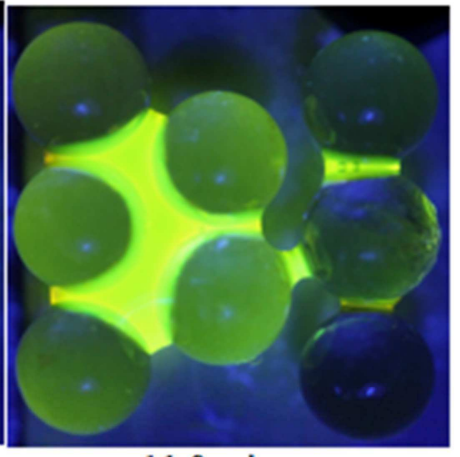

$11.0 \mathrm{~min}$

Fig. 1. Evolution of the water body between 8 glass spheres subjected to evaporation at constant temperature, constant ambient vapor pressure. The red arrow indicates a localized non-symmetric unstable mode of the interface evolution (air entry finger).

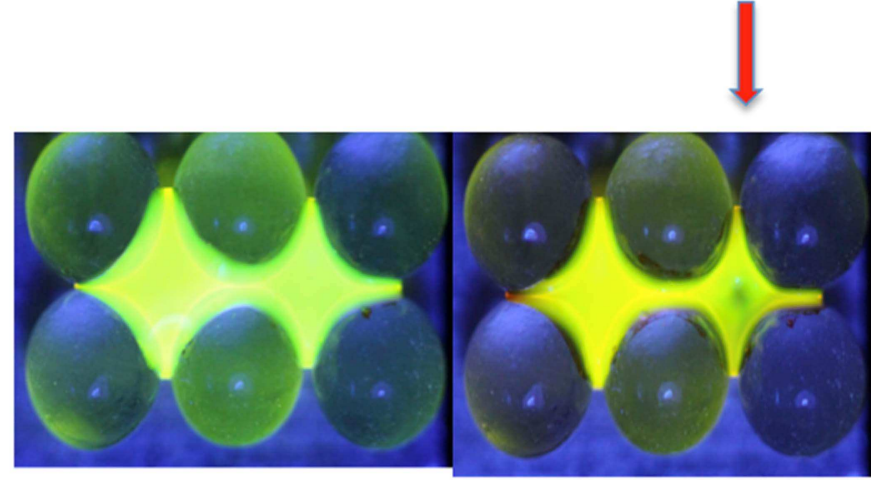

$0.0 \mathrm{~min}$

$26.0 \mathrm{~min}$

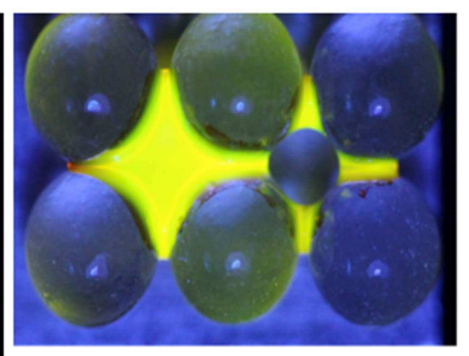

$27.0 \mathrm{~min}$

Fig. 2. Evolution of the water body between 6 glass spheres subjected to evaporation at constant temperature, constant ambient vapor pressure. The red arrow indicates a symmetric localized unstable mode of the interface evolution.

\section{MODELING}

As mentioned earlier, modeling of desiccation cracking via macroscopic approach suffers from a major handicap. A simple consideration of even 1D drying of a constrained saturated granular body easily shows that a modest total stress is generated in reaction to the constraints and that it is tensile, whereas the suction induced by the evaporation flux is significant. Consequently, the resulting effective stress appears compressive. This is counterintuitive, as drying failure evidently occurs through tensile cracking.

To solve this enigma, Scherer (1992) postulated a very elegant scenario, originally idea for drying silica gels suggesting that desiccation cracking is necessarily triggered through crack propagation from a tip of a preexisting structural flaw, at which a substantial total stress concentration occurs. As a result, the remote total 
stress is substantially magnified through the stress concentration and becomes larger in absolute terms than the elevated suction. As a result the local effective stress becomes tensile, and hence prone to reach the tensile strength of the material.

Based on the presented results we postulate that the non-symmetric finger of air entry as described above, constitutes a sufficient imperfection (flaw) in the drying granular body to produce a concentration of total stress induced by the kinematic restraints to the drying shrinkage around the tip of the flaw, to generate crack propagation, as proposed by Péron et al., 2010, and Hu et al., 2011, following the idea of Scherer, 1992.

In this spirit we consider an air finger (flaw) with depth $c$ and curvature radius at the finger tip of $r_{c}$ at the soil surface, which is surrounded by the micro-tubes pore system as shown in Fig.3. The system of micro-tubes representing at the meso-scale the pore system is adapted from the model of $\mathrm{Hu}$ et al., 2012a to simulate the shrinkage mechanism for silty soil due to water evaporation. In this model, the soil pore structure is approximated by a network of hollow cylinder tubes. The external radius $\left(R_{\text {ext }}\right)$ of the tubes is considered equal to $3.6 \mu \mathrm{m}$ and the internal radius $\left(R_{i n t}\right)$ is equal to $0.75 \mu \mathrm{m}$. These radii are obtained based on the mercury intrusion porosimetry data on Bioley silt (Hu et al., 2012a). Using the principles of linear fracture mechanics, the stress at the external boundary of the tubes, which are placed near the flaw tip can be calculated. Following the methodology proposed by Scherer (1992), the far-field (macro-scale) stress at crack initiation can be found as (stress is positive in tension)

$$
\sigma_{x}=\frac{K_{I c r i t}+\frac{1}{2} p \sqrt{\pi r_{c}}}{B \sqrt{\pi c}}
$$

where $K_{I \text { crit }}$ is the critical stress intensity factor, $B$ is a constant and equal to 1.12 and $p$ is the fluid pressure inside of the pore.

The depth of a flaw $(c)$ can be estimated from Fig. 1c as 5 times the diameter of the biggest pore, assessed for Bioley silt as $d=1.5 \mu \mathrm{m}$ (hence $c=7.5 \mu \mathrm{m}$ for that silt). The flaw tip curvature radius $r_{c}$ is found to be of the same order as the internal radius of the biggest pore $(d / 2=0.375 \mu \mathrm{m})$. A desiccation crack is generally initiated when the soil suction reaches the suction value at the air entry moment. Considering the biggest pore size of $1.5 \mu \mathrm{m}$, the suction value at air entry for Bioley silt has been calculated as $100 \mathrm{kPa}$ (Péron, 2008, Péron et al., 2009). Adopting a value of $0.16 \mathrm{kN} / \mathrm{m}^{3 / 2}$ for the critical stress intensity factor as measured by Chertkov (1995) for a sandy loam soil, a value of $\sigma_{x}=39 \mathrm{kPa}$ is obtained for the macro scale total stress from Eq.(1). This value is close enough to the one obtained experimentally by Péron (2008) in simulations of the drying experiments on constrained slabs of Bioley silt $(25 \mathrm{kPa})$. The local total stress at the flaw tip is hence estimated as

$$
\sigma_{c}^{\max }=2 B \sigma_{x} \sqrt{\frac{c}{r_{c}}}
$$

which yields a value of $395 \mathrm{kPa}$ (tension). 


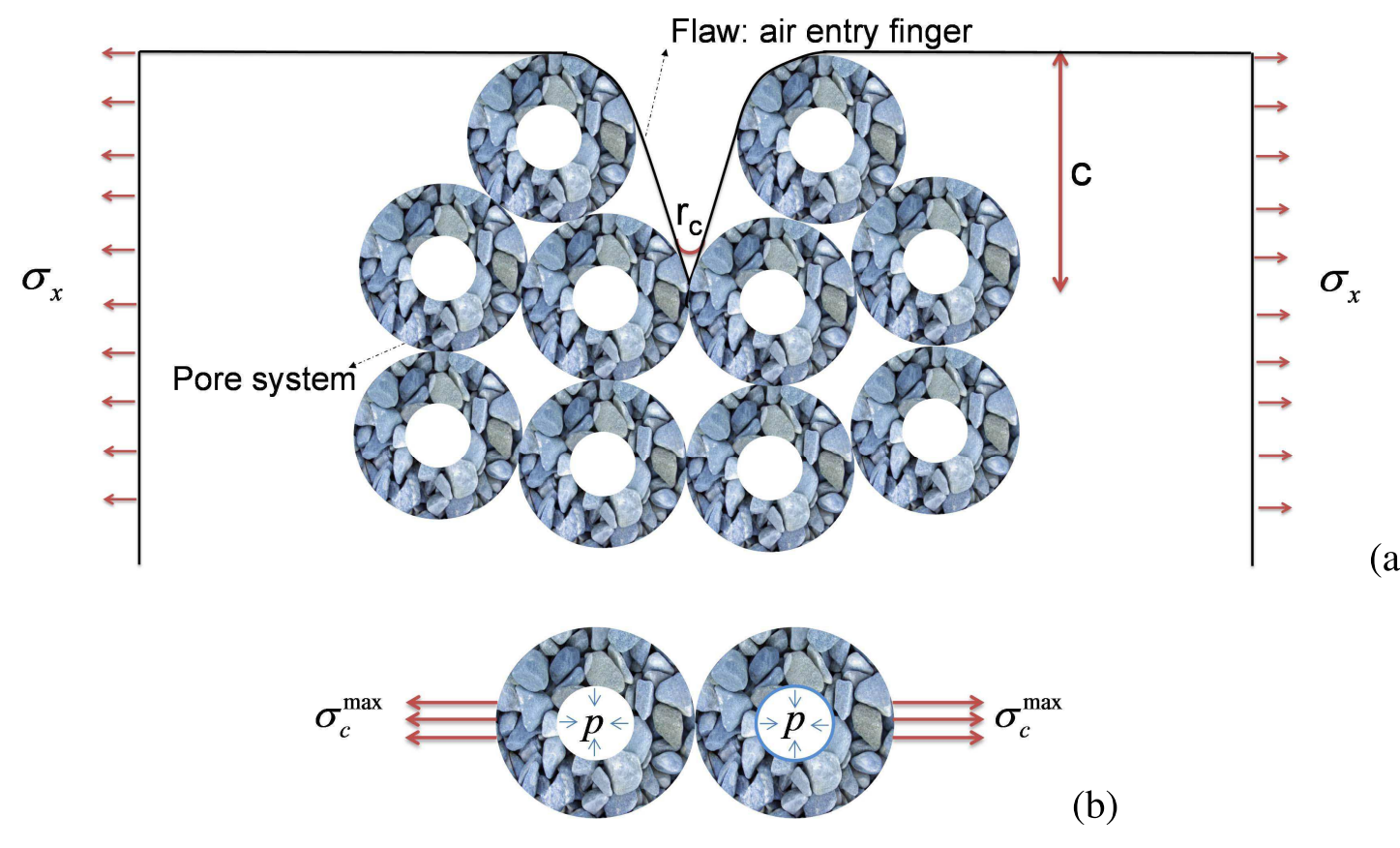

(a)

\section{Fig. 3. a) Idealized meso-scale pore network in the vicinity of a air finger; b) Micro-scale stress state for the two tubes in the vicinity of the flaw tip for the purpose of FE analysis at the micro-scale.}

We shall now evaluate the stress resulting within the tubes imposed by the conditions arising at the tip, as calculated at the meso-scale. For that purpose we employ a finite element representation of the two tubes at the micro-scale. The tubes are located near the air finger tip in an ideally symmetrical position as shown in Fig. $3 \mathrm{~b}$. The material of the tubes is assumed as linear elastic, with $E=52 \mathrm{MPa}$ and $v=0.3$ following Péron's (2008) data for Bioley silt. The total stress at the crack tip $\left(\sigma_{c}^{\max }\right)$ is applied on the contact boundary of the tubes and the tubes are considered to be restricted at their bottoms and free at their tops. The Z_Soil.PC (Zace Service Ltd) finite element code was used for numerical modelling of the problem and Fig. $4 \mathrm{a}$ and $\mathrm{b}$ shows the modelling of the maximum and minimum principal stresses in the tubes for a contact area of $\frac{R_{e x t} \pi}{10}$ respectively. As shown the maximum total principal stress is equal to $607 \mathrm{kPa}$ and it occurs at the contact area between the tubes (upper corner of the contact area at the left hand side of the tube Fig. 4). The possible failure mechanism is a Mode I failure (tensile) in this zone. Once two tubes are separated by tensile failure the length of the flaw increases to $c+2 R_{e x t}$ and a crack propagates through the next layer of the tubes.

As shown via the numerical simulations, the effective principal stress at failure is equal to $507 \mathrm{kPa}$ (tension), however at the macro-scale this value is $-61 \mathrm{kPa}$ (compression). These differences in sign of the effective stress explains the apparent contradiction of obvious soil shrinkage during the drying process due to compressive effective stress at macro-scale and observing the desiccation cracks which originate from microscale by a tensile failure mechanism. 


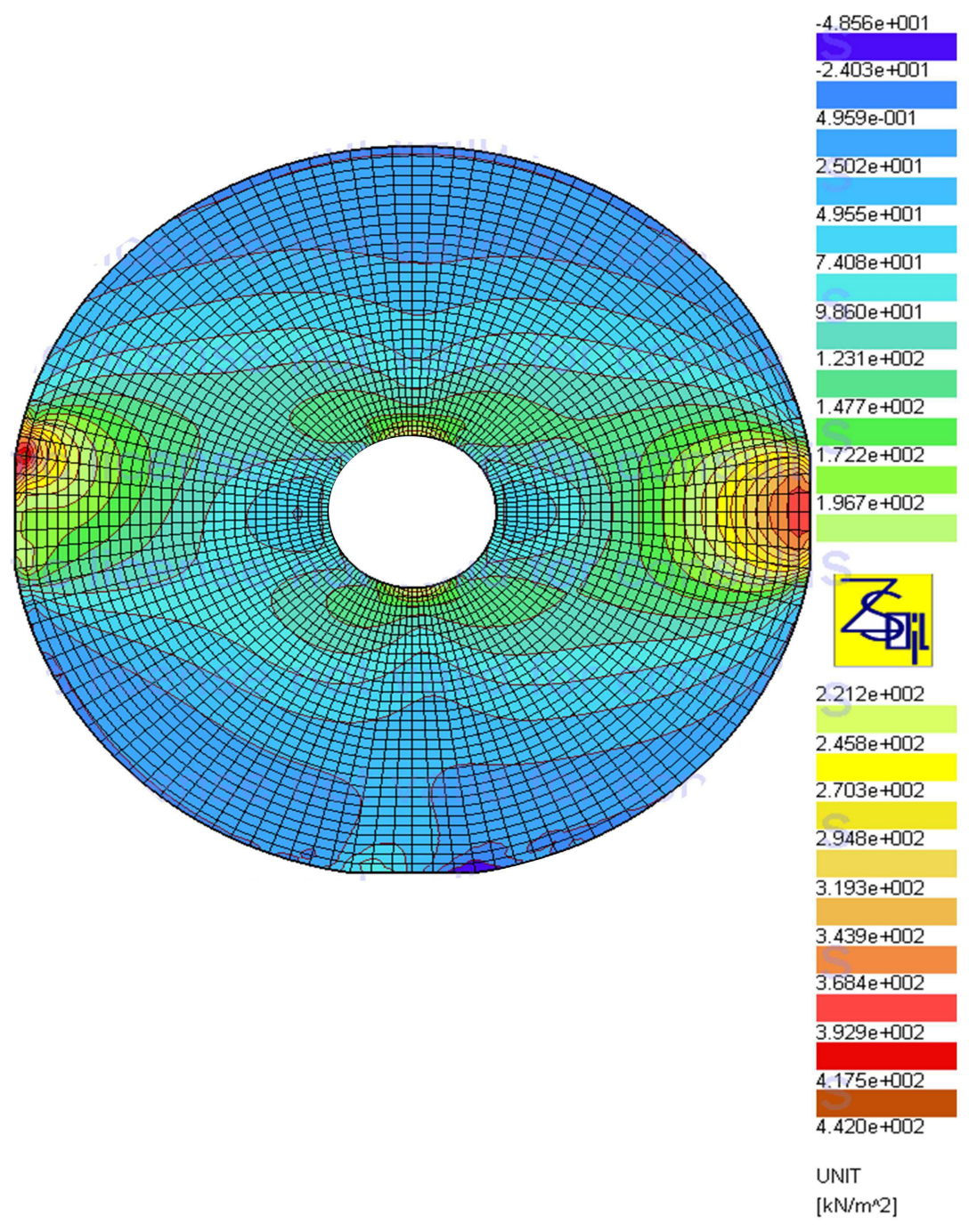

Fig. 4: Maximum principal stress within one pore surroundings.

\section{CONCLUSIONS}

The purpose of the paper is to assess whether air entry fingers may constitute sufficient imperfections in the soil mass to be the cause of a stress concentration and hence result into sufficiently high local effective stress at the air finger tip and provoke crack propagation at the site of the air entry. Both meso-scale linear fracture mechanics analysis and micro-scale finite element analysis yield the stress values in the plausible range. The presented analysis must be viewed as a feasibility study, rather than a technical modeling exercise. 


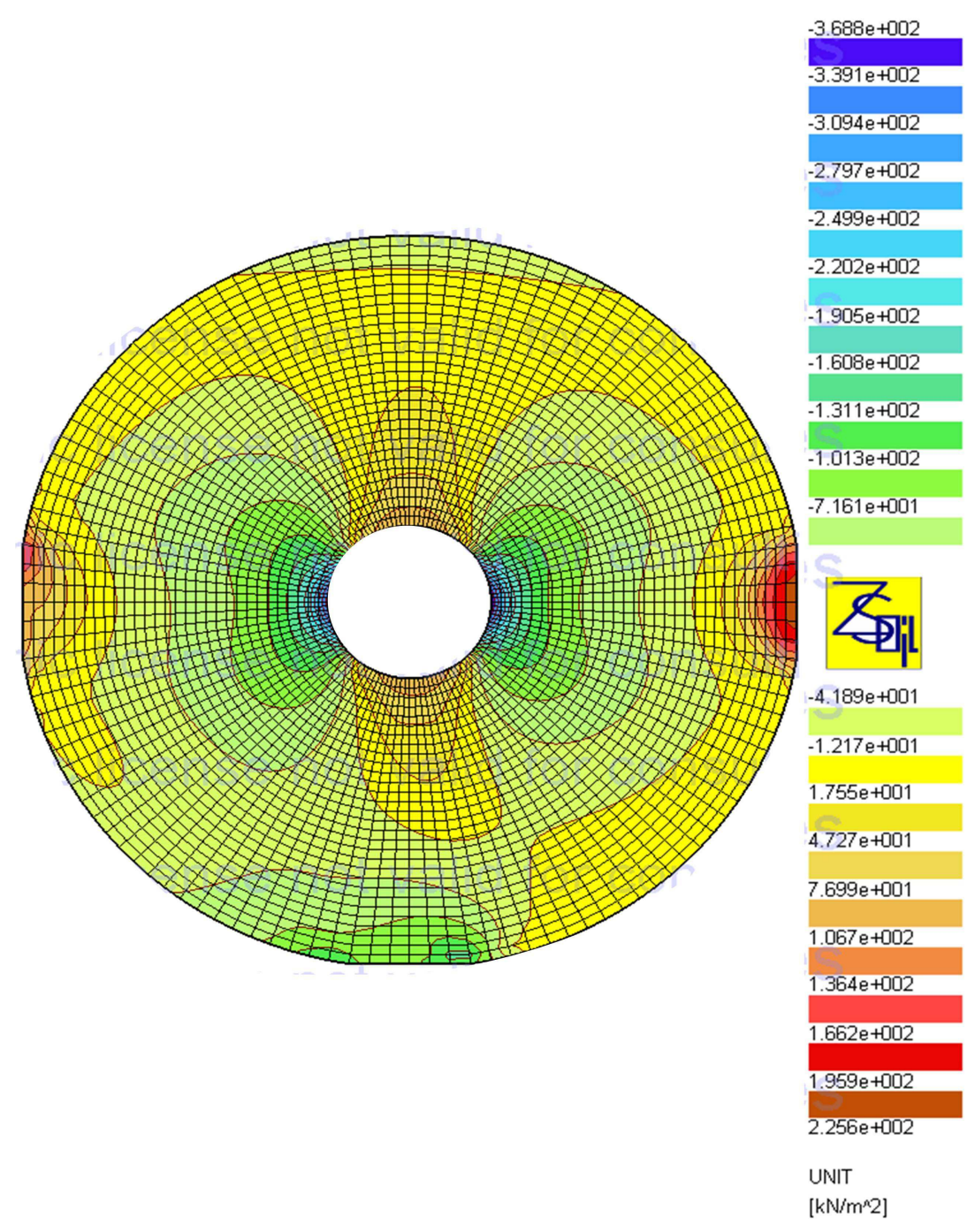

Fig. 5: Minimum principal stress within one pore space surrounding.

\section{ACKNOWLEDGMENTS}

This work was partially funded by the US National Science Foundation, grant \#0700294 of CMMI Division, Geomechanics and Geomaterials Program and the Swiss National Science Foundation, grant 200021_124702. 


\section{REFERENCES}

Chertkov, V.Y., (1995), Evaluation for soil of crack net connectedness and critical stress intensity factor, Int. Agrophysics, 9, 189 -195

Hu, L.B., H. Péron, L. Laloui and T. Hueckel (2011), A multi-scale multi-physics model of soil drying, GEOFRONTIERS 2011

Hu, L. B., H. Péron, T. Hueckel, L. Laloui, (2012) Desiccation shrinkage of non-clayey soils: multi-physics mechanisms and a microstructural model, Int. J. Numer. Anal. Meth. Geomech, DOI: 10.1002/nag.2108.

Hu, L. B., H. Péron, T. Hueckel, L. Laloui, (2012) Desiccation shrinkage of non-clayey soils: a numerical study, Int. J. Numer. Anal. Meth. Geomech., DOI: 10.1002/nag.2107

Mielniczuk, B., T. Hueckel, M. S. El Youssoufi (2013) Micro-scale study of rupture in desiccating granular media, GEOCONGRESS 2013

H. Péron, T. Hueckel, L. Laloui, L.B. Hu (2009) Fundamentals of desiccation cracking of fine-grained soils: experimental characterisation and mechanisms identification, Canadian Geotechnical J., 46, 1177-1201

Péron, H., L. Laloui, L.B. Hu, and T. Hueckel (2010), Desiccation of drying soils, in Mechanics of Unsaturated Geomaterials, p. 55-86, edited by L. Laloui, J. Wiley, Hoboken, NJ,

Péron, H. (2008) Desiccation cracking of soils, PhD thesis, EPFL, Lausanne

Scherer G.W., Crack-tip stress in gels, J. Non-Cryst. Solids, vol. 144, 1992, p. 210-216 